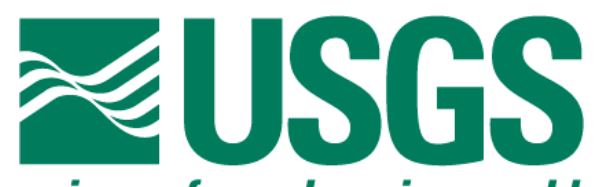

science for a changing world

\title{
Preliminary Analytical Results for Ash and Burned Soils from the October 2007 Southern California Wildfires
}

By Geoffrey S. Plumlee, Deborah A. Martin, Todd Hoefen, Raymond Kokaly, Philip Hageman, Alison Eckberg, Gregory P. Meeker, Monique Adams, Michael Anthony, and Paul J. Lamothe

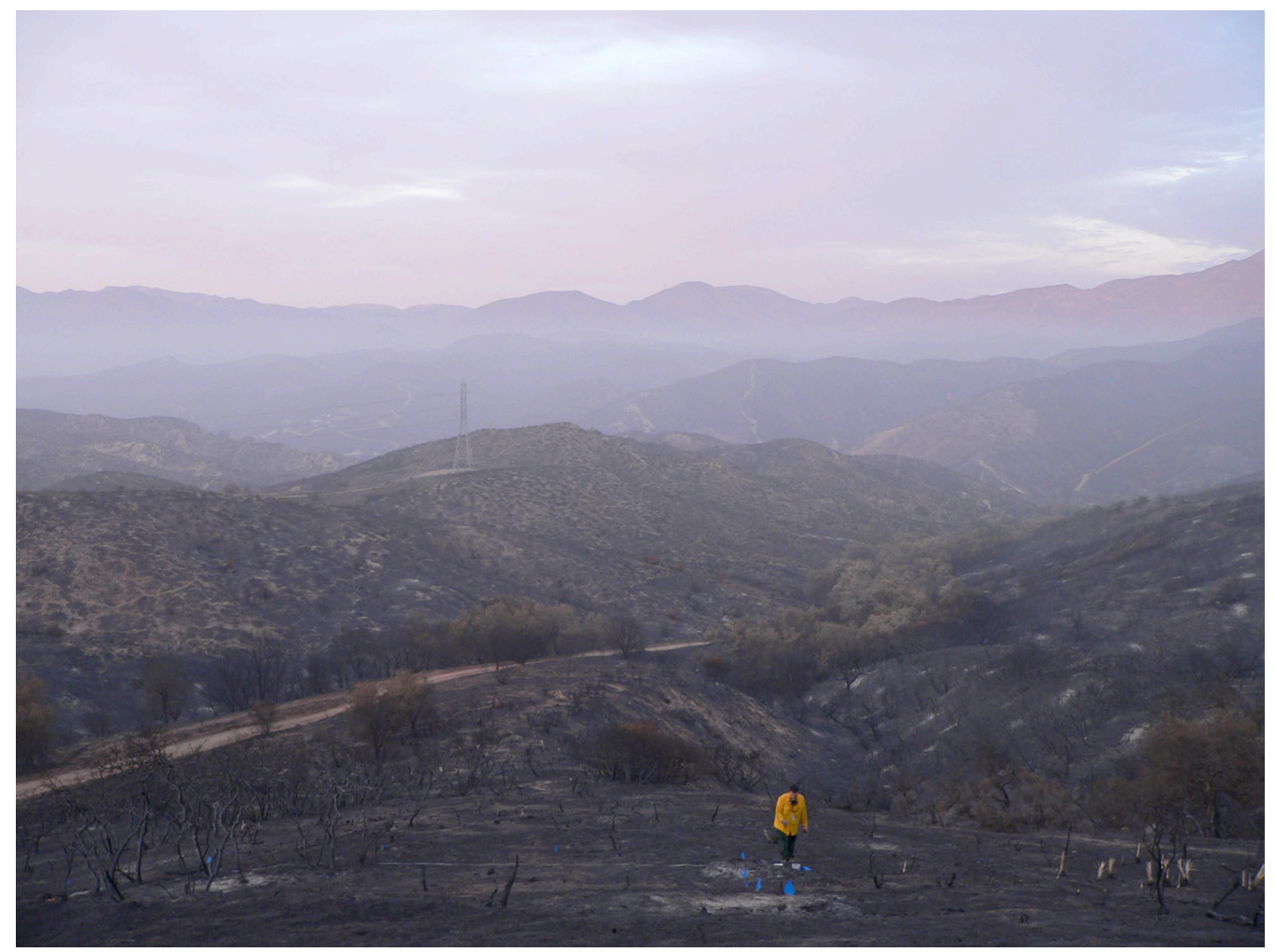

Open-File Report 2007-1407

U.S. Department of the Interior

U.S. Geological Survey 


\section{U.S. Department of the Interior DIRK KEMPTHORNE, Secretary}

\section{U.S. Geological Survey \\ Mark D. Myers, Director}

U.S. Geological Survey, Reston, Virginia 2007

For product and ordering information:

World Wide Web: http://www.usgs.gov/pubprod

Telephone: 1-888-ASK-USGS

For more information on the USGS - the Federal source for science about the Earth,

its natural and living resources, natural hazards, and the environment:

World Wide Web: http://www.usgs.gov

Telephone: 1-888-ASK-USGS

Suggested citation:

Plumlee, G.S., Martin, D.A., Hoefen, T., Kokaly, R., Hageman, P., Eckberg, A., Meeker, G.P., Adams, M., Anthony, M., and Lamothe, P.J., 2007, Preliminary analytical results for ash and burned soils from the 0 ctober 2007 southern California Wildfires: U.S. Geological Survey Open-File Report 2007-1407.

Any use of trade, product, or firm names is for descriptive purposes only and does not imply endorsement by the U.S. Government.

Although this report is in the public domain, permission must be secured from the individual copyright owners to reproduce any copyrighted material contained within this report. 


\section{Overview}

The U.S. Geological Survey (USGS) collected ash and burned soils from about 28 sites in southern California wildfire areas (Harris, Witch, Ammo, Santiago, Canyon and Grass Valley) from Nov. 2 through 9, 2007 (table 1). USGS researchers are applying a wide variety of analytical methods to these samples, with the goal of helping identify characteristics of the ash and soils from wildland and suburban burned areas that may be of concern for their potential to adversely affect water quality, human health, endangered species, and debris-flow or flooding hazards. These studies are part of the Southern California Multi-Hazards Demonstration Project, and preliminary findings are presented here.

\section{Sampling and Sample Preparation}

Most samples were collected from wildland burn areas and typically include separate samples of ash and underlying burned soil. The goal of the wildland sampling was to understand potential differences in ash and soil characteristics that may result from differences in the type of burned vegetation and underlying bedrock geology. At a given site, sampling of ash and underlying soil was done by the collection and compositing of multiple subsamples at evenly spaced intervals along a transect or on spokes radiating from a centroid; such sampling has been found to produce the most statistically valid representation of spatially heterogeneous materials such as mine wastes (Smith and others, 2000).

Partly in response to a request from a Burned Area Emergency Rehabilitation (BAER) Team, USGS scientists collected composite samples of ash/debris and underlying soil from two burned residential areas within the Grass Valley and Harris burns. Each of these two composite residential samples consists of multiple random grab subsamples collected within or near multiple burned residences.

The USGS also recently acquired hyperspectral remote sensing data with the HyMap imaging spectrometer (Cocks and others, 1998) over several of the burned areas. The detailed chemical and physical properties of the ash and soil samples will be linked to the remote sensing data using field and laboratory reflectance measurements of the samples, resulting in maps that extend estimates of ash impact on environment and health across the burned areas.

Samples were shipped to Denver overnight. Samples were homogenized prior to splitting. Most sample analysis types are being conducted on a split that was obtained by cone-and-quartering of the full sample, and that was then sieved to less than $2 \mathrm{~mm}$ to remove coarse particles.

Ash analyses completed to date have focused on the residential ash and burned soil samples, and to a lesser extent on wildland samples from the same burned areas. This initial focus is in response to the BAER team request to provide information on burned residences within the Grass Valley fire area. Additional analyses are intended on wildland ash and soil samples from other burned areas, pending funding availability. 


\section{Analytical Methods}

Analytical methods (Taggart, 2002) performed to date on a subset of the samples include:

(1) Bulk chemical composition on a ground $<2 \mathrm{~mm}$ split using inductively coupled plasma-mass spectrometry (ICP-MS) for a 44-element suite. The analysis follows 4-acid digestion of the sample; this digestion typically is considered a total digestion of most environmental materials.

(2) Deionized water leach test on an unground $<2 \mathrm{~mm}$ split, to simulate interactions of ash with rainfall (Hageman, 2007). One part ash is added to 20 parts deionized water, the mixture shaken for 5 minutes, and the leachate is then filtered and analyzed for $\mathrm{pH}$, alkalinity, conductivity, anions by ion chromatography, cations and metals by ICP-MS, and mercury by continuous flow injection-cold vaporatomic fluorescence spectrometry (AFS).

(3) Particle characterization using scanning electron microscopy (SEM)

Samples analyses currently in process include: X-ray diffraction for mineralogy; bioaccessibility of metals and metalloids in simulated biofluids; and, particle size distribution. If additional funding is secured, samples will also be analyzed for organic contaminants, microbial characteristics, and total amounts of and forms of carbon, sulfur, and nitrogen.

\section{Preliminary Findings}

Preliminary analyses of the ash and soil samples indicate several features of potential environmental or health concern.

(1) Water leach tests (table 2) show that the residential ash samples generate high $\mathrm{pH}$ levels (12.5-12.7). These levels suggest that ash from burned residences can generate caustic alkalinity when it comes into contact with rainwater or waterbased body fluids (such as perspiration or fluids lining the respiratory tract). In contrast, similar water leachates of the limited number of wildland ash samples analyzed to date generate somewhat less caustic alkalinity and lower $\mathrm{pH}(9.8-$ 10.9). USGS water leach tests on ash from vegetation combusted in the laboratory at various controlled temperatures indicate that leachate $\mathrm{pH}$ correlates with temperature of combustion, with leachate $\mathrm{pH}$ maximizing at 12.5 for combustion temperatures over $600^{\circ} \mathrm{C}$ (J. Crock, unpub. data). Accordingly, the higher $\mathrm{pH}$ and alkalinity of the residential ash leachates compared to wildland ash leachates might indicate that residential fires burned at higher temperatures than did fires in wildland areas; however, the combustion of diverse man-made materials in residences cannot be ruled out as a possible source for the high residential leachate $\mathrm{pH}$ values.

The caustic alkalinity of exposed residential and wildland ash may diminish over time as it interacts with and is neutralized by rainwater and atmospheric carbon dioxide. However, further work is needed to determine the rate at which this 
neutralization may occur. High-alkalinity rainwater runoff from burned areas may also become neutralized as it mixes with and is diluted by sufficient quantities of near-neutral $\mathrm{pH}$ fresh water from unburned areas.

(2) Compared to average US soils, the two residential ash samples can contain elevated arsenic (up to 140 parts per million, $\mathrm{ppm}$ or $\mathrm{mg} / \mathrm{kg}$ ), lead (up to $344 \mathrm{ppm}$ ), antimony (up to $32 \mathrm{ppm}$ ), copper (up to 1,370 ppm), or zinc (up to 2,800 ppm) (table 3). For arsenic, lead, and antimony, concentrations in one or both of the residential composite ash samples approach or exceed U.S. Environmental Protection Agency residential soil Preliminary Remediation Goals (PRGs: 0.4$0.62 \mathrm{ppm}$ arsenic; $31 \mathrm{ppm}$ antimony, 150-400 ppm lead) (EPA, 2004). Chromium levels are also elevated in the residential ash (up to $354 \mathrm{ppm}$ ); further analyses are underway to determine what proportion of the chromium in the residential ash is the more toxic hexavalent form. The EPA PRGs are "...tools for evaluating and cleaning up contaminated sites. They are risk-based concentrations that are intended to assist risk assessors and others in initial screening-level evaluations of environmental measurements." (EPA, 2004). Although these PRGs were not designed for wildfire ash, they do provide one possible measure against which the chemical composition of the wildfire ash and burned soils can be preliminarily compared.

The water leach tests performed to date suggest that most of these metals have relatively low solubility under the conditions of this particular leach test; however, further tests are needed, as element solubilities may vary with differences in solid:water ratio. Results of pending physiologically based bioaccessibility tests using simulated gastrointestinal fluids and simulated lung fluids will provide insights into the potential bioaccessibility of these metals and metalloids along ingestion and inhalation pathways.

(3) The BAER team request for sampling and analysis of residences included a request to evaluate whether asbestos is present in or absent from the residential ash. Reconnaissance SEM analyses completed to date have not conclusively identified the presence of asbestos in the residential ash samples. Because the makeup of ash may vary substantially throughout a burned residence, and due to both the reconnaissance nature of our sampling within each residence and the reconnaissance nature of the SEM analyses of the collected samples, the negative finding to date for asbestos does not guarantee that asbestos is absent from the burned residences; it might be present locally in areas of the residences not sampled or analyzed. As noted by CALEPA (2003), potentially higher levels of asbestos (and lead) might be expected in burned residential areas with houses constructed earlier than the early 1980s, when use of asbestos and leaded paints in construction materials was common. 


\section{Guidance from the Findings}

These USGS findings are consistent with the scientific knowledge about wildfire ash that has led the California Department of Health Services (CDHS, 2003), California EPA (CALEPA, 2003), various California counties (i.e., San Diego County, 2007), and $\mathrm{NIOSH}(2007$, p. 6$)$ to issue advisories regarding appropriate precautionary measures that should be followed by persons working in burned wildland areas or cleaning up burned residences to avoid possible health problems associated with ash exposure. Such measures include, for example, use of appropriate respiratory protection, gloves, longsleeved shirts and long pants, dust mitigation measures, and washing of skin contacted by the ash.

The USGS results further indicate that rainwater runoff from burned residential areas may be a source of high-alkalinity waters with possibly elevated concentrations of some metals or metalloids of health or environmental concern. Given the extent of the burned residential areas, these results suggest that residential portions of burned watersheds may need enhanced cleanup attention before the rains, and possibly enhanced monitoring during the rains. Rainwater runoff from the burned residential and wildland areas may also adversely affect water quality and critical species habitat in receiving streams by causing spikes in alkalinity, concentrations of some metals, and levels of some nutrients, as has been noted in burned areas elsewhere in the United States (Surber, 2002; Meixner, 2004).

The results also indicate that more detailed characterization of the ash and debris may be warranted within residential areas. In particular, more detailed characterization will aid in the development of appropriate disposal strategies for materials removed from burned residences that minimize potential metal release from the materials into landfills or the environment.

Results of analyses in progress will be released as they become available.

\section{Needed Additional Studies}

The focus of the current study was primarily burned wildland areas. It is important to note that the two residential samples collected are not representative of all residential areas that burned in southern California. Therefore, additional studies are recommended in burned residential areas to fully examine the potential environmental and health issues associated with residential ash and burned soils. Needed studies include, for example (1) variability of ash composition within and between residences in a given neighborhood and (2) variability in ash composition as a function of residence age, type, and setting (such as north- or south-facing slope, proximity to other residences, intensity and duration of fire, and type of construction).

\section{Acknowledgments}

Robert N. Fisher, Carlton Rochester, Eric Reichard, Greg Mendez, and other scientists from the USGS California Water Science Center office in San Diego provided invaluable scientific input, logistical support, and other assistance for the field sampling. Heather Lowers provided technical assistance with the SEM studies, and David Walters 
helped immensely with sample processing. James Crock, Dennis Eberl, and other scientists provided very helpful technical reviews on all or portions of the report.

\section{USGS Contacts}

Geoffrey S. Plumlee, gplumlee@usgs.gov

Deborah A. Martin, damartin@usgs.gov

Todd Hoefen (field sampling), thoefen@usgs.gov

Ray Kokaly (field sampling and remote sensing), raymond@usgs.gov

Phil Hageman (leach tests), phageman@usgs.gov

Greg Meeker (SEM analysis), gmeeker@usgs.gov

Paul Lamothe (analytical chemistry), plamothe@usgs.gov

\section{References}

CALEPA, 2003, Safe cleanup of fire ash: http://www.calepa.ca.gov/Disaster/Fire2003/FireAsh.pdf

CDHS, 2003, State health director offers advice on safe cleanup of fire ash: California Dept. of Health Services Press Release 03-89, http://www.applications.dhs.ca.gov/pressreleases/store/PressReleases/03-89.html

Cocks, T., Jenssen, R., Stewart, A., Wilson, I., and Shields, T., 1998, The HyMap airborne hyperspectral sensor: The system, calibration and performance, in Proceedings of the 1st EARSEL Workshop on Imaging Spectroscopy, Zurich.

EPA, 2004, U.S. Environmental Protection Agency, Region 9, Preliminary remediation goals, http://www.epa.gov/region09/waste/sfund/prg/index.html - prgtable.

Hageman, P.L., 2007, U.S. Geological Survey field leach test for assessing water reactivity and leaching potential of mine wastes, soils, and other geologic and environmental materials: U.S. Geological Survey Techniques and Methods Report 5D3, http://pubs.usgs.gov/tm/2007/05D03/pdf/TM5-D3 508.pdf

Meixner, T., 2004, Wildfire impacts on water quality: Southwest Hydrology, Sept./Oct. 2004, p. 23-24, http://www.swhydro.arizona.edu/archive/V3_N5/featurette7.pdf

NIOSH, 2003, Worker safety during fire cleanup: National Institutes of Occupational Safety and Health Fact Sheet, 7 p., http://emergency.cdc.gov/disasters/wildfires/pdf/firecleanupworkers.pdf

San Diego County, 2007, Post-fire health tips and ash and debris cleanup guidance. http://www.sdcountyrecovery.com/documents/AshDebrisPublicHealthTipsCleanup1108-07.pdf 
Smith, K.S., and Huyck, H.L.O., 1999, An overview of the abundance, relative mobility, bioavailability, and human toxicity of metals, Chapter 2, in Plumlee, G.S., and Logsdon, M.J., eds., The environmental geochemistry of mineral deposits, Part A: Processes, techniques, and health issues: Reviews in Economic Geology, v. 6A: Littleton, Colorado, Society of Economic Geologists, Inc., p. 29-70.

Smith, K.S., Ramsey, C.A., and Hageman, P.L., 2000, Sampling strategy for the rapid screening of mine-waste dumps on abandoned mine lands, in ICARD 2000:

Proceedings from the Fifth International Conference on Acid Rock Drainage, Denver, Colorado, May 21-24, 2000: Society for Mining, Metallurgy, and Exploration, Inc., p. $1,453-1,461$.

Surber, G., 2002, Section 3, water quality concerns after wildfire, in Knight, J.E., ed., After wildfire-Information for landowners coping with the aftermath of wildfire: Montana State University Extension Service, p. 9-14, http://www.montana.edu/wwwpb/pubs/445503water.pdf

Taggart, J.E., Jr., ed., 2002, Analytical methods for chemical analysis of geologic and other materials: U.S. Geological Survey Open-File Report 02-0223, http:/pubs.usgs.gov/of/2002/ofr-02-0223/ 
Table 1. Locations and types of samples collected in this study.

\begin{tabular}{|c|c|c|c|c|}
\hline SAMPLE ID & Latitude & Longitude & Sampling Type & Location Type \\
\hline Harris01 & $32^{\circ} 37^{\prime} 53.90^{\prime \prime}$ & $116^{\circ} 52^{\prime} 04.08^{\prime \prime}$ & Spoke & Hilltop \\
\hline Harris02W,S,A & $32^{\circ} 35^{\prime} 46.3^{\prime \prime}$ & $116^{\circ} 46^{\prime} 05.7^{\prime \prime}$ & Opportunistic & Hillside \\
\hline Harris03A,B,C & $32^{\circ} 35^{\prime} 46.3^{\prime \prime}$ & $116^{\circ} 46^{\prime} 05.7^{\prime \prime}$ & Opportunistic & Tailings pile \\
\hline Harris04 & $32^{\circ} 42^{\prime} 07.0^{\prime \prime}$ & $116^{\circ} 57^{\prime} 36.9^{\prime \prime}$ & Spoke & Hillside near Sweetwater \\
\hline Harris05 & $32^{\circ} 42^{\prime} 11.0^{\prime \prime}$ & $116^{\circ} 57^{\prime} 39.8^{\prime \prime}$ & Spoke & Drainage basin near Sweetwater \\
\hline Harris06 & $32^{\circ} 39^{\prime} 47.3^{\prime \prime}$ & $116^{\circ} 40^{\prime} 51.2^{\prime \prime}$ & Transect & Steep hillside \\
\hline Harris 07 & $32^{\circ} 37^{\prime} 22.2^{\prime \prime}$ & $116^{\circ} 41^{\prime} 26.6^{\prime \prime}$ & Random sampling & Residential, mobile homes \\
\hline Witch01 & $33^{\circ} 09^{\prime} 09.13^{\prime \prime}$ & $116^{\circ} 47^{\prime} 00.91^{\prime \prime}$ & Transect & Steep hillside \\
\hline Witch02 & $33^{\circ} 07^{\prime} 11.74^{\prime \prime}$ & $116^{\circ} 4727.11^{\prime \prime}$ & Transect & Hillside \\
\hline Witch03 & $33^{\circ} 06^{\prime} 49.4^{\prime \prime}$ & $116^{\circ} 49^{\prime} 01.4^{\prime \prime}$ & Grab & Roadcut \\
\hline Witch04 & $33^{\circ} 06^{\prime} 25.3^{\prime \prime}$ & $116^{\circ} 49^{\prime} 37.3^{\prime \prime}$ & Transect & Hilltop \\
\hline Witch05 & $33^{\circ} 05^{\prime} 2.5^{\prime \prime}$ & $116^{\circ} 59^{\prime} 18.9^{\prime \prime}$ & Transect & Dried streambed \\
\hline Santiago01 & $33^{\circ} 43^{\prime} 12.0^{\prime \prime}$ & $117^{0} 35^{\prime} 54.6^{\prime \prime}$ & Transect & Sampled streambed from this point then east 150 yards \\
\hline Santiago02 & $33^{\circ} 43^{\prime} 12.0^{\prime \prime}$ & $117^{0} 35^{\prime} 54.6^{\prime \prime}$ & Transect & Sampling started and went west 140 yards in streambed \\
\hline Santiago03 & $33^{\circ} 43^{\prime} 12.0^{\prime \prime}$ & $117^{0} 35^{\prime} 54.6^{\prime \prime}$ & Transect & Sampling started and went west 140 yards on hillside \\
\hline Santiago04 & $33^{\circ} 43^{\prime} 11.4^{\prime \prime}$ & $117^{0} 35^{\prime} 59.6^{\prime \prime}$ & Spoke & Next to dried streambed \\
\hline Santiago 05 & $33^{\circ} 43^{\prime} 03.01^{\prime \prime}$ & $117^{\circ} 36^{\prime} 37.8^{\prime \prime}$ & Spoke & Next to dried streambed \\
\hline Santiago06 & $33^{\circ} 42^{\prime} 59.1^{\prime \prime}$ & $117^{0} 37^{\prime} 13.2^{\prime \prime}$ & Spoke & Broad valley bottom \\
\hline Santiago07 & $33^{\circ} 43^{\prime} 04.2^{\prime \prime}$ & $117^{\circ} 37^{\prime} 15.0^{\prime \prime}$ & Spoke & Drainage draining into Santiago 06 from the north \\
\hline Santiago08 & $33^{\circ} 43^{\prime} 02.0^{\prime \prime}$ & $117^{\circ} 39^{\prime} 34.6^{\prime \prime}$ & Spoke & Hillside \\
\hline Santiago09 & $33^{\circ} 43^{\prime} 02.0^{\prime \prime}$ & $117^{\circ} 39^{\prime} 34.6^{\prime \prime}$ & Grab & Potential hydrophobic soil \\
\hline Ammo1 & $33^{\circ} 22^{\prime} 52.3^{\prime \prime}$ & $117^{\circ} 32^{\prime} 55.4^{\prime \prime}$ & Spoke & Marine base hillside \\
\hline Canyon01 & $34^{\circ} 02^{\prime} 44.0^{\prime \prime}$ & $118^{\circ} 42^{\prime} 16.8^{\prime \prime}$ & Transect & Very steep hillside near Pepperdine U. \\
\hline Canyon02 & $34^{\circ} 3^{\prime} 53.0^{\prime \prime}$ & $118^{\circ} 41^{\prime} 50.0^{\prime \prime}$ & Transect & Steep hillside \\
\hline Canyon03 & $34^{\circ} 3^{\prime} 45.5^{\prime \prime}$ & $118^{\circ} 41^{\prime} 50.1$ & Transect & Steep Hillside \\
\hline Canyon04 & $34^{\circ} 01^{\prime} 57.7^{\prime \prime}$ & $118^{\circ} 42^{\prime} 04.1^{\prime \prime}$ & Transect & Hillside near beach \\
\hline GrassValley01 & $34^{\circ} 16^{\prime} 02.6^{\prime \prime}$ & $117^{0} 13^{\prime} 06.0^{\prime \prime}$ & Random Sampling & Modern homes \\
\hline GrassValley02 & $34^{\circ} 16^{\prime} 06.3^{\prime \prime}$ & $117^{0} 13^{\prime} 06.6^{\prime \prime}$ & Spoke & Hillside \\
\hline
\end{tabular}


Table 2. Composition of water leachates of California wildfire ash and burned soil samples. Fluoride, chloride, and nitrate by ion chromatography; cations, metals and sulfate by ICP-MS; mercury by AFS. Analytical results are not blank subtracted. nr — not reported, nm—not measured.

\begin{tabular}{|c|c|c|c|c|c|c|c|c|}
\hline Sample & $\mathrm{pH}$ & $\begin{array}{c}\text { Specific } \\
\text { Conductance } \\
(\mu \mathrm{S} / \mathrm{cm})\end{array}$ & $F(\mathrm{mg} / \mathrm{L})$ & $\mathrm{Cl}(\mathrm{mg} / \mathrm{L})$ & $\begin{array}{c}\mathrm{NO}_{3} \\
(\mathrm{mg} / \mathrm{L})\end{array}$ & $\operatorname{Ag}(\mu \mathrm{g} / \mathrm{L})$ & $\mathrm{Al}(\mu \mathrm{g} / \mathrm{L})$ & As $(\mu \mathrm{g} / \mathrm{L})$ \\
\hline Harris 06 Ash & 10.9 & 890 & 4.3 & 32 & 4.1 & $<1$ & 252 & 1 \\
\hline Harris 06 Ash Duplicate & 10.8 & 890 & 4 & 31.6 & 4.11 & $<1$ & 235 & 2 \\
\hline Grass Valley 02 Soil & 8.2 & 140 & 3.2 & 1.2 & 0.9 & $<1$ & 292 & 1 \\
\hline Grass Valley 02 Ash & 9.8 & 360 & 1.9 & 5.4 & 1.8 & $<1$ & 4540 & 1 \\
\hline Grass Valley 01 Soil & 10.9 & 460 & 0.7 & 8.1 & 2 & $<1$ & 1570 & 2 \\
\hline Grass Valley 01 Ash & 12.7 & 5040 & $<.08$ & 70 & $<.08$ & $<1$ & 73.2 & 2 \\
\hline Grass Valley 01 Ash (ICP-MS dup) & $\mathrm{nm}$ & $\mathrm{nm}$ & $\mathrm{nm}$ & $\mathrm{nm}$ & $\mathrm{nm}$ & $<1$ & 75.1 & 1 \\
\hline Harris 07 Soil & 10.6 & 240 & 1.2 & 25.3 & 1 & $<1$ & 975 & 219 \\
\hline Harris 07 Ash & 12.5 & 3950 & 3 & 285 & 13.5 & $<1$ & 3650 & 13.5 \\
\hline Blank & 6 & 0.77 & $<.08$ & $<.08$ & $<.08$ & $<1$ & 3.8 & $<1$ \\
\hline
\end{tabular}

\begin{tabular}{|c|c|c|c|c|c|c|c|c|}
\hline Sample & $\mathrm{Ba}(\mu \mathrm{g} / \mathrm{L})$ & $\mathrm{Be}(\mu \mathrm{g} / \mathrm{L})$ & $\mathrm{Bi}(\mu \mathrm{g} / \mathrm{L})$ & $\begin{array}{c}\mathrm{Ca} \\
(\mathrm{mg} / \mathrm{L})\end{array}$ & $\mathrm{Cd}(\mu \mathrm{g} / \mathrm{L})$ & $\mathrm{Ce}(\mu \mathrm{g} / \mathrm{L})$ & Co $(\mu \mathrm{g} / \mathrm{L})$ & $\mathrm{Cr}(\mu \mathrm{g} / \mathrm{L})$ \\
\hline Harris 06 Ash & 109 & $<0.05$ & $<0.2$ & 21.2 & 0.03 & $<0.01$ & 0.33 & 1.5 \\
\hline Harris 06 Ash Duplicate & 116 & $<0.05$ & $<0.2$ & 25.0 & 0.04 & $<0.01$ & 0.38 & 1.5 \\
\hline Grass Valley 02 Soil & 29.3 & $<0.05$ & $<0.2$ & 20.5 & $<0.02$ & 0.54 & 0.43 & $<1$ \\
\hline Grass Valley 02 Ash & 254 & $<0.05$ & $<0.2$ & 41.2 & $<0.02$ & $<0.01$ & 0.24 & 1.4 \\
\hline Grass Valley 01 Soil & 21.3 & $<0.05$ & $<0.2$ & 63.2 & $<0.02$ & 0.03 & 0.10 & 7.7 \\
\hline Grass Valley 01 Ash & 158 & $<0.05$ & 0.21 & 668 & 0.05 & $<0.01$ & 0.28 & 31.9 \\
\hline Grass Valley 01 Ash (ICP-MS dup) & 153 & 0.06 & $<0.2$ & 629 & 0.06 & $<0.01$ & 0.48 & 32.7 \\
\hline Harris 07 Soil & 28.3 & $<0.05$ & $<0.2$ & 24.5 & 0.04 & 0.08 & 0.24 & 3.0 \\
\hline Harris 07 Ash & 1670 & $<0.05$ & $<0.2$ & 427 & 0.06 & $<0.01$ & $<0.02$ & 32.4 \\
\hline Blank & 0.41 & $<0.05$ & $<0.2$ & $<0.2$ & $<0.02$ & $<0.01$ & $<0.02$ & $<1$ \\
\hline
\end{tabular}


Table 2-Continued.

\begin{tabular}{|c|c|c|c|c|c|c|c|c|}
\hline Sample & Cs $(\mu \mathrm{g} / \mathrm{L})$ & $\mathrm{Cu}(\mu \mathrm{g} / \mathrm{L})$ & Dy $(\mu \mathrm{g} / \mathrm{L})$ & $\operatorname{Er}(\mu \mathrm{g} / \mathrm{L})$ & $\mathrm{Eu}(\mu \mathrm{g} / \mathrm{L})$ & $\mathrm{Fe}(\mu \mathrm{g} / \mathrm{L})$ & $\mathrm{Ga}(\mu \mathrm{g} / \mathrm{L})$ & $G d(\mu g / L)$ \\
\hline Harris 06 Ash & 0.24 & 23.9 & $<0.005$ & $<0.005$ & 0.02 & $<50$ & 0.68 & $<0.005$ \\
\hline Harris 06 Ash Duplicate & 0.25 & 23.3 & $<0.005$ & $<0.005$ & 0.02 & $<50$ & 0.60 & $<0.005$ \\
\hline Grass Valley 02 Soil & $<0.02$ & 2.6 & 0.04 & 0.02 & 0.02 & 50 & 0.31 & 0.04 \\
\hline Grass Valley 02 Ash & 0.05 & 4.5 & $<0.005$ & $<0.005$ & 0.03 & $<50$ & 1.7 & $<0.005$ \\
\hline Grass Valley 01 Soil & $<0.02$ & 2.8 & $<0.005$ & $<0.005$ & $<0.005$ & $<50$ & 1.3 & 0.005 \\
\hline Grass Valley 01 Ash & 0.32 & 8.9 & $<0.005$ & $<0.005$ & 0.02 & $<50$ & 0.38 & $<0.005$ \\
\hline Grass Valley 01 Ash (ICP-MS dup) & 0.32 & 10.0 & 0.009 & $<0.005$ & 0.03 & $<50$ & 0.39 & $<0.005$ \\
\hline Harris 07 Soil & $<0.02$ & 10.4 & 0.01 & $<0.005$ & 0.005 & $<50$ & 0.96 & 0.009 \\
\hline Harris 07 Ash & 0.89 & 8.7 & $<0.005$ & $<0.005$ & 0.21 & $<50$ & 4.5 & $<0.005$ \\
\hline Blank & $<0.02$ & $<0.5$ & $<0.005$ & $<0.005$ & $<0.005$ & $<50$ & $<0.05$ & 0.005 \\
\hline Sample & $\mathrm{Ge}(\mu \mathrm{g} / \mathrm{L})$ & $\mathrm{Hg}(\mathrm{ng} / \mathrm{L})$ & $\mathrm{Ho}_{0}(\mu \mathrm{g} / \mathrm{L})$ & $\mathrm{K}(\mathrm{mg} / \mathrm{L})$ & $\mathrm{La}(\mu \mathrm{g} / \mathrm{L})$ & $\mathrm{Li}(\mu \mathrm{g} / \mathrm{L})$ & $\operatorname{Lu}(\mu \mathrm{g} / \mathrm{L})$ & $\underset{(\mathrm{mg} / \mathrm{L})}{\mathrm{Mg}}$ \\
\hline Harris 06 Ash & 0.07 & 25 & $<0.005$ & $\mathrm{nr}$ & $<0.01$ & 12.4 & $<0.1$ & 7.13 \\
\hline Harris 06 Ash Duplicate & 0.08 & 21 & $<0.005$ & $\mathrm{nr}$ & $<0.01$ & 13.1 & $<0.1$ & 10.7 \\
\hline Grass Valley 02 Soil & $<0.05$ & 6 & 0.007 & 3.55 & 0.28 & $<0.1$ & $<0.1$ & 1.38 \\
\hline Grass Valley 02 Ash & 0.06 & 8 & $<0.005$ & 14.6 & $<0.01$ & 20.9 & $<0.1$ & 6.05 \\
\hline Grass Valley 01 Soil & $<0.05$ & $<5$ & $<0.005$ & 5.99 & 0.02 & 0.4 & $<0.1$ & 0.37 \\
\hline Grass Valley 01 Ash & $<0.05$ & $<5$ & $<0.005$ & 14.3 & $<0.01$ & 12.4 & $<0.1$ & 0.02 \\
\hline Grass Valley 01 Ash (ICP-MS dup) & $<0.05$ & $\mathrm{~nm}$ & $<0.005$ & 14.2 & $<0.01$ & 13.5 & $<0.1$ & 0.04 \\
\hline Harris 07 Soil & 0.09 & $<5$ & $<0.005$ & 3.32 & 0.04 & 5.9 & $<0.1$ & 0.85 \\
\hline Harris 07 Ash & $<0.05$ & $<5$ & $<0.005$ & 41.6 & 0.01 & 41.3 & $<0.1$ & 0.03 \\
\hline Blank & $<0.05$ & $<5$ & $<0.005$ & 0.06 & $<0.01$ & 0.6 & $<0.1$ & $<0.01$ \\
\hline
\end{tabular}


Table 2-Continued.

\begin{tabular}{|c|c|c|c|c|c|c|c|c|}
\hline Sample & $M n(\mu g / L)$ & Mo $(\mu \mathrm{g} / \mathrm{L})$ & $\begin{array}{c}\mathrm{Na} \\
(\mathrm{mg} / \mathrm{L})\end{array}$ & $\begin{array}{c}N b \\
(\mu \mathrm{g} / \mathrm{L})\end{array}$ & $\begin{array}{c}N d \\
(\mu \mathrm{g} / \mathrm{L})\end{array}$ & $\mathrm{Ni}(\mu \mathrm{g} / \mathrm{L})$ & $P(\mathrm{mg} / \mathrm{L})$ & $\mathrm{Pb}(\mu \mathrm{g} / \mathrm{L})$ \\
\hline Harris 06 Ash & 2.6 & 21.4 & 8.91 & 0.72 & $<0.01$ & 2.0 & 0.1 & 0.2 \\
\hline Harris 06 Ash Duplicate & 3.1 & 18.7 & 9.66 & $<0.2$ & $<0.01$ & 2.0 & 0.1 & 0.3 \\
\hline Grass Valley 02 Soil & 172 & $<2$ & 0.54 & 0.20 & 0.23 & 1.5 & 0.3 & 0.5 \\
\hline Grass Valley 02 Ash & 16.9 & 10.1 & 0.84 & $<0.2$ & $<0.01$ & 2.1 & 0.02 & 0.3 \\
\hline Grass Valley 01 Soil & 1.3 & 3.0 & 6.98 & $<0.2$ & 0.01 & 0.8 & 0.02 & 0.4 \\
\hline Grass Valley 01 Ash & 0.3 & 19.5 & 25.7 & 0.31 & $<0.01$ & 7.1 & $<0.01$ & 157 \\
\hline Grass Valley 01 Ash (ICP-MS dup) & 0.4 & 18.7 & 25.4 & $<0.2$ & $<0.01$ & 11.5 & $<0.01$ & 163 \\
\hline Harris 07 Soil & 4.1 & 4.6 & 9.10 & $<0.2$ & 0.04 & 1.4 & 0.1 & 0.56 \\
\hline Harris 07 Ash & 0.4 & 26.7 & 97.4 & $<0.2$ & $<0.01$ & 2.9 & $<0.01$ & 37.8 \\
\hline Blank & 0.3 & $<2$ & 0.26 & $<0.2$ & $<0.01$ & $<0.4$ & $<0.01$ & 0.2 \\
\hline Sample & $\operatorname{Pr}(\mu \mathrm{g} / \mathrm{L})$ & $\operatorname{Rb}(\mu \mathrm{g} / \mathrm{L})$ & $\mathrm{Sb}(\mu \mathrm{g} / \mathrm{L})$ & $S c(\mu \mathrm{g} / \mathrm{L})$ & Se $(\mu \mathrm{g} / \mathrm{L})$ & $\underset{\text { (m g/L) }}{\mathrm{SiO}}$ & $\underset{(\mu \mathrm{g} / \mathrm{L})}{S \mathrm{~m}}$ & $\underset{\mathrm{SO4}}{\mathrm{SOg} / \mathrm{L})}$ \\
\hline Harris 06 Ash & $<0.01$ & 49.6 & 8.61 & 1.1 & 7.2 & 7.8 & $<0.01$ & 170 \\
\hline Harris 06 Ash Duplicate & $<0.01$ & 49.6 & 6.88 & 0.9 & 7.3 & 7.9 & $<0.01$ & 188 \\
\hline Grass Valley 02 Soil & 0.06 & 2.94 & 4.84 & $<0.6$ & $<1$ & 0.9 & 0.04 & 8 \\
\hline Grass Valley 02 Ash & $<0.01$ & 9.22 & 7.15 & $<0.6$ & 3.1 & 1.1 & $<0.01$ & 105 \\
\hline Grass Valley 01 Soil & $<0.01$ & 2.30 & 9.89 & $<0.6$ & 1.6 & 1.8 & $<0.01$ & 187 \\
\hline Grass Valley 01 Ash & $<0.01$ & 20.8 & 3.07 & $<0.6$ & 3.6 & 1.8 & $<0.01$ & 986 \\
\hline Grass Valley 01 Ash (ICP-MS dup) & $<0.01$ & 21.2 & 2.43 & 0.8 & 3.4 & 1.8 & $<0.01$ & 941 \\
\hline Harris 07 Soil & $<0.01$ & 1.74 & 27.5 & $<0.6$ & 2.7 & 3.2 & $<0.01$ & 18 \\
\hline Harris 07 Ash & $<0.01$ & 59.2 & 4.10 & $<0.6$ & 6.3 & 3.1 & $<0.01$ & 107 \\
\hline Blank & $<0.01$ & 0.03 & 1.07 & $<0.6$ & $<1$ & $<0.2$ & $<0.01$ & $<2$ \\
\hline
\end{tabular}


Table 2-Continued.

\begin{tabular}{|c|c|c|c|c|c|c|c|c|}
\hline Sample & $\operatorname{Sr}(\mu \mathrm{g} / \mathrm{L})$ & $\mathrm{Ta}(\mu \mathrm{g} / \mathrm{L})$ & $\mathrm{Tb}(\mu \mathrm{g} / \mathrm{L})$ & $\operatorname{Th}(\mu \mathrm{g} / \mathrm{L})$ & $\mathrm{Ti}(\mu \mathrm{g} / \mathrm{L})$ & $\mathrm{TI}(\mu \mathrm{g} / \mathrm{L})$ & $\begin{array}{c}\mathrm{Tm} \\
(\mu \mathrm{g} / \mathrm{L})\end{array}$ & $U(\mu g / L)$ \\
\hline Harris 06 Ash & 218 & 0.85 & $<0.005$ & $<0.2$ & 2.8 & $<0.1$ & $<0.005$ & $<0.1$ \\
\hline Harris 06 Ash Duplicate & 232 & 0.23 & $<0.005$ & $<0.2$ & 2.5 & $<0.1$ & $<0.005$ & 0.10 \\
\hline Grass Valley 02 Soil & 198 & 0.42 & 0.006 & $<0.2$ & 2.7 & $<0.1$ & $<0.005$ & 0.47 \\
\hline Grass Valley 02 Ash & 797 & 0.20 & $<0.005$ & $<0.2$ & 1.7 & $<0.1$ & $<0.005$ & 1.04 \\
\hline Grass Valley 01 Soil & 236 & 0.05 & $<0.005$ & $<0.2$ & 2.9 & $<0.1$ & $<0.005$ & $<0.1$ \\
\hline Grass Valley 01 Ash & 1160 & 0.28 & $<0.005$ & $<0.2$ & 13.5 & $<0.1$ & $<0.005$ & $<0.1$ \\
\hline Grass Valley 01 Ash (ICP-MS dup) & 1160 & 0.34 & $<0.005$ & $<0.2$ & 13.6 & $<0.1$ & $<0.005$ & $<0.1$ \\
\hline Harris 07 Soil & 69.5 & 0.1 & $<0.005$ & $<0.2$ & 2.6 & $<0.1$ & $<0.005$ & 0.32 \\
\hline Harris 07 Ash & 748 & $<0.02$ & $<0.005$ & $<0.2$ & 1.7 & $<0.1$ & $<0.005$ & $<0.1$ \\
\hline Blank & $<0.5$ & $<0.02$ & $<0.005$ & $<0.2$ & $<0.5$ & $<0.1$ & $<0.005$ & $<0.1$ \\
\hline
\end{tabular}

\begin{tabular}{lrrrrr} 
Sample & $V(\mu \mathrm{g} / \mathrm{L})$ & $W(\mu \mathrm{g} / \mathrm{L})$ & $\mathrm{Y}(\mu \mathrm{g} / \mathrm{L})$ & $\mathrm{Yb}(\mu \mathrm{g} / \mathrm{L})$ & $\mathrm{Zn}(\mu \mathrm{g} / \mathrm{L})$ \\
\hline Harris 06 Ash & 39.2 & 1.88 & 0.01 & 0.005 & 3.6 \\
Harris 06 Ash Duplicate & 33.3 & 1.43 & 0.01 & 0.005 & 2.9 \\
Grass Valley 02 Soil & 3.6 & $<0.5$ & 0.22 & 0.01 & 5.2 \\
Grass Valley 02 Ash & 6.8 & 1.93 & 0.07 & $<0.005$ & 3.3 \\
Grass Valley 01 Soil & 16.4 & $<0.5$ & 0.01 & $<0.005$ & 1.9 \\
Grass Valley 01 Ash & 10.0 & 0.62 & 0.07 & $<0.005$ & 154 \\
Grass Valley 01 Ash (ICP-MS dup) & 10.1 & 0.50 & 0.01 & 0.01 & 168 \\
Harris 07 Soil & 16.9 & 0.87 & 0.04 & 0.008 & 6.1 \\
Harris 07 Ash & 4.0 & 2.43 & 0.09 & $<0.005$ & 71.4 \\
Blank & $<0.5$ & $<0.5$ & $<0.01$ & 0.005 & 9.4
\end{tabular}


Table 3. Bulk chemical composition of selected California wildfire ash and burned soil samples, as determined by ICP-MS. EPA residential soil preliminary remediation goals vary for chromium, depending upon the proportion of hexavalent chromium (VI) compared to trivalent chromium (III) present.

\begin{tabular}{|c|c|c|c|c|c|c|c|c|c|}
\hline Sample & $\mathrm{Ag} \mathrm{ppm}$ & Al ppm & As ppm & Ba ppm & Be ppm & Bi ppm & Ca ppm & Cd ppm & Ce ppm \\
\hline Detection Limit & $<1$ & $<50$ & $<1$ & $<0.2$ & $<0.03$ & $<0.06$ & $<100$ & $<0.007$ & $<0.1$ \\
\hline Harris07-soil & 3.4 & 80400 & 49.4 & 660 & 1.3 & 0.40 & 29300 & 2.1 & 43.1 \\
\hline Harris07-ash & 8.5 & 76000 & 140 & 858 & 0.92 & 0.54 & 130000 & 4.8 & 35.2 \\
\hline Grass Valley-01soil & $<1$ & 93200 & 4.0 & 892 & 2.1 & 0.13 & 42400 & 0.16 & 85.0 \\
\hline Grass Valley-01ash & 0.96 & 40800 & 17.9 & 541 & 1.0 & 0.27 & 191000 & 1.0 & 29.4 \\
\hline $\begin{array}{l}\text { EPA Region IX residential soil } \\
\text { preliminary remediation goals } \\
\text { (EPA, 2004) }\end{array}$ & 390 & 76000 & $0.39-0.62$ & 5400 & 150 & & & 37 & \\
\hline $\begin{array}{l}\text { Western US soil mean (Smith } \\
\text { and Huyck, 1999). * US mean. }\end{array}$ & $0.05^{*}$ & 58000 & 5.5 & 580 & 0.68 & & 18000 & $0.06^{*}$ & 65 \\
\hline Sample & Co ppm & Cr ppm & Cs ppm & Cu ppm & Fe ppm & Ga ppm & K ppm & La ppm & Li ppm \\
\hline Detection Limit & $<0.03$ & $<0.5$ & $<0.003$ & $<2$ & $<50$ & $<0.02$ & $<20$ & $<0.05$ & $<0.3$ \\
\hline Harris07-soil & 11.2 & 55.9 & 2.0 & 471 & 40400 & 17.4 & 16800 & 23.3 & 14.8 \\
\hline Harris07-ash & 12.2 & 91.1 & 1.8 & 1120 & 23600 & 15.9 & 16300 & 19.8 & 19.0 \\
\hline Grass Valley-01soil & 17.2 & 175 & 3.4 & 404 & 49900 & 21.7 & 23100 & 54.6 & 33.9 \\
\hline Grass Valley-01ash & 27.3 & 354 & 1.4 & 1370 & 51200 & 8.9 & 11400 & 16.9 & 14.2 \\
\hline $\begin{array}{l}\text { EPA Region IX residential soil } \\
\text { preliminary remediation goals } \\
\text { (EPA, 2004) }\end{array}$ & 900 & $\begin{array}{r}210(1 / 6 \mathrm{VI} / \mathrm{III}), \\
30 \text { (all VI), } 100000 \\
\text { (all III) }\end{array}$ & & 3100 & 23000 & & & & 1600 \\
\hline $\begin{array}{l}\text { Western US soil mean (Smith } \\
\text { and Huyck, 1999). * US mean. }\end{array}$ & 7.1 & 41 & $6^{*}$ & 21 & 21000 & 16 & 18000 & 30 & 22 \\
\hline
\end{tabular}


Table 3-Continued.

\begin{tabular}{|c|c|c|c|c|c|c|c|c|c|c|}
\hline Sample & Mg ppm & Mn ppm & Mo ppm & $\mathrm{Na} \mathrm{ppm}$ & $\mathrm{Nb} p \mathrm{pm}$ & $\mathrm{Ni} p \mathrm{pm}$ & P ppm & $\mathrm{Pb} p \mathrm{pm}$ & Rb ppm & Sb ppm \\
\hline Detection Limit & $<6$ & $<0.7$ & $<0.05$ & $<20$ & $<0.1$ & $<0.3$ & $<5$ & $<0.4$ & $<0.01$ & $<0.04$ \\
\hline Harris07-soil & 10200 & 727 & 0.86 & 26600 & 10 & 14.0 & 1080 & 62.9 & 58.9 & 8.6 \\
\hline Harris07-ash & 10800 & 774 & 3.5 & 21300 & 12 & 41.6 & 1540 & 192 & 58.3 & 31.5 \\
\hline Grass Valley-01soil & 15400 & 867 & 8.3 & 12700 & 26 & 14.1 & 1030 & 162 & 122 & 5.1 \\
\hline Grass Valley-01ash & 7840 & 396 & 73.0 & 9360 & 10 & 36.6 & 509 & 344 & 47.2 & 24.9 \\
\hline
\end{tabular}

EPA Region IX residential

soil preliminary remediation

goals (EPA, 2004)

1800

390

1600

$150-400$

Western US soil mean

(Smith and Huyck, 1999).

*US mean.

7400

380

0.85

9700

8.7

15

320

17

69

0.47

\begin{tabular}{l}
$\quad$ Sample \\
\hline Detection Limit \\
Harris07-soil \\
Harris07-ash \\
Grass Valley-01soil \\
Grass Valley-01ash \\
EPA Region IX residential \\
soil preliminary \\
remediation goals (EPA, \\
2004)
\end{tabular}

$\begin{array}{rr}\text { Sc ppm } & \text { Sr ppm } \\ <0.04 & <0.1\end{array}$

$\begin{array}{crr} & \text { Th ppm } & \text { Ti ppm } \\ <0.8 & <0.1 & \end{array}$

$<40$

$<0.08$

U ppm

$\mathrm{V} p \mathrm{pm}$

\begin{tabular}{rrr}
$r$ pm & \multicolumn{1}{c}{ Y ppm } & \multicolumn{1}{c}{ Zn ppm } \\
$<0.2$ & $<0.05$ & $<3$ \\
100 & 41.1 & 458 \\
67.8 & 26.0 & 2220 \\
127 & 29.4 & 211 \\
54.0 & 15.5 & 2800
\end{tabular}

$\begin{array}{lll}10.4 & 277 & 5.76\end{array}$

$13.7-398 \quad 931$

5120

$0.36<213$

2.13

2.23

$\begin{array}{ll}0.38 & 2.23 \\ 0.70 & 3.15\end{array}$

6730

0.32

1.89

54.0

2800

remediation goals (EPA

Western US soil mean

(Smith and Huyck, 1999).

*US mean.

200

9.1

2200

5.2

16

78

23000

4000

2.5

70

22

55 